\title{
Role of Portal Vein Embolization in Hepatocellular Carcinoma Management and Its Effect on Recurrence: A Case-control Study
}

\author{
Rohan C. Siriwardana $\cdot$ Chung Mau Lo • \\ See Ching Chan $\cdot$ Sheung Tat Fan
}

Published online: 13 March 2012

(c) The Author(s) 2012. This article is published with open access at Springerlink.com

\begin{abstract}
Background Liver regeneration that occurs after portal vein embolization (PVE) may have adverse effects on the microscopic tumor foci in the residual liver mass in patients with hepatocellular carcinoma (HCC).

Methods Fifty-four HCC patients with inadequate functional residual liver volume were offered PVE during a seven-year period. Among them, 34 (63\%) patients underwent curative resection. They were compared with a matched control group $(n=102)$ who underwent surgery without PVE. Postoperative complications, pattern of recurrence, and survival were compared between groups.

Results In the PVE group, a pre-embolization functional residual liver volume of $23 \%$ (12-33.5\%) improved to $34 \%$ $(20-54 \%)(p=0.005)$ at the time of surgery. When the two groups were compared, minor (PVE, 24\%; control, $29 \% ; p=0.651$ ) and major (PVE, 18\%; control, 15\%; $p=0.784$ ) complications were similar. After a follow-up period of 35 months (standard deviation 25 months), extrahepatic recurrences were detected in 10 PVE patients (29\%) and 41 control patients $(40 \%)(p=0.310)$. Intrahepatic recurrences were seen in $10(29 \%)$ and 47 (46\%) cases $(p=0.109)$ in the PVE and control groups,
\end{abstract}

The abstract of this article was presented at the 21st Conference of the Asian Pacific Association for the Study of the Liver, Bangkok, Thailand, 17-20 February 2011.

R. C. Siriwardana - C. M. Lo $(\varangle) \cdot$ S. C. Chan · S. T. Fan Department of Surgery, The University of Hong Kong, 102 Pokfulam Road, Hong Kong, People's Republic of China e-mail: chungmlo@hkucc.hku.hk

C. M. Lo - S. C. Chan · S. T. Fan

State Key Laboratory for Liver Research, The University of Hong Kong, 102 Pokfulam Road, Hong Kong, People's Republic of China respectively. In the PVE group, 41\% $(n=14)$ of the recurrences were detected before one year, compared with $42 \%(n=43)$ in the control group $(p=1)$. Disease-free survival rates at 1,3 , and 5 years were 57,29 , and $26 \%$ in the control group and 60, 42, and $42 \%$ in the PVE group (log-rank, $p=0.335$ ). On multivariate analysis, PVE was not a factor affecting survival $(p=0.821)$.

Conclusions Portal vein embolization increases the resectability of initially unresectable $\mathrm{HCC}$ due to inadequate functional residual liver volume, and it has no deleterious oncological effect after major resection of HCC.

\section{Introduction}

Surgery is the treatment of choice for patients with hepatocellular carcinoma (HCC) [1]. Yet not all detected HCCs are amenable to surgical resection although the resection rate varies from center to center. A functional residual liver volume (FRLV) in excess of $25 \%$ in a normal liver or $40 \%$ in a diseased liver is considered necessary to avoid postoperative liver failure [2]. For patients with marginal FRLV, portal vein embolization (PVE) increases the resectability of tumor by a corresponding increase of the contralateral side.

Portal vein embolization for patients with $\mathrm{HCC}$ raises special considerations. The effectiveness and safety of the procedure in the presence of background diseased liver and progression of the primary tumor are major concerns. Furthermore, PVE and subsequent resection may promote microscopic tumor foci. Some consider that the same growth factors are accountable for hepatic regeneration following PVE, but others contradict this possibility and suggest that different mechanisms may be involved [3, 4]. 
Wakabayashi [5], for instance, has reported an increase in extrahepatic tumor recurrence following PVE, while Tanaka et al. [6] has documented increased overall survival in a group of 33 patients. The present study evaluated the short-term and long-term outcomes of resection of HCC in patients who had preoperative PVE against a matched control group of patients who did not have preoperative PVE.

\section{Patients and methods}

The study included 54 consecutive HCC patients with inadequate FRLV who had undergone PVE during the period 2002-2009. Eleven (20\%) of them underwent percutaneous PVE, whereas the others $(80 \%)$ were treated with an open procedure via the ileocolic vein. The patients were reassessed after an interval of 4-6 weeks in terms of liver function, tumor progression, and improvement in liver volume. Resection was considered feasible for patients with FRLV more than $20 \%$ of a normal liver, $30 \%$ of a fibrotic liver, and $40 \%$ of a cirrhotic liver. Patients with liver function test results comparable to pre-embolization results and those who had no evidence of disease progression also proceeded to surgery. Transarterial chemoembolization (TACE) was performed after an interval of two weeks in cases of larger tumors or when additional time was considered necessary for liver regeneration.

Resection was considered not to be feasible for 20 patients. They were offered other forms of therapy on an individual basis. These included radiofrequency ablation, high-intensity focused ultrasound ablation, and repeated cycles of TACE. One patient underwent liver transplantation outside our center. The median overall survival in this group was 10 months (range: 8-11 months). Overall, 34 $(63 \%)$ patients who had improved FRLV underwent surgery after a median interval of 45 days (range: 2696 days).

Thirty-four patients (the PVE group) who had undergone resection were compared with a matched control sample. The controls were selected by screening a database of patients who underwent surgery as the first treatment. For each case in the PVE group, three controls were selected, matching the year of surgery, type of resection [7] (grouped according to Brisbane 2000 terminology of liver anatomy and resections), and the presence of vascular permeation.

Postoperative complications in the two groups were categorized according to the Clavien grading [8] for further analysis, with grades 1 and 2 complications being considered minor and grades 3 and 4 considered major.

All patients were regularly followed up at the outpatient clinic and were prospectively monitored for recurrence.
The standard protocol of surveillance included contrastenhanced computed tomography (CT) scan at one month after resection, followed by liver function test, serum alpha-fetoprotein level check, ultrasonography or CT scan, and chest radiograph every three months. Suspected intrahepatic recurrence was confirmed by hepatic angiography, post-lipiodol CT scan and, if necessary, percutaneous needle biopsy.

The two groups were compared in terms of their baseline clinicopathological features (Table 1). On univariate analysis, there was a significant difference in the preoperative platelet count $(p=0.030)$, international normalized ratio $(p=0.035)$, and resection margin $(p=0.023)$ between the two groups. Furthermore, 16 patients in the PVE group underwent concurrent TACE, but none of the patients in the control group were subjected to this procedure $(p<0.001)$. There was no significant difference in other factors, including maximum tumor size, number of tumor nodules, presence of microsatellite nodules, differentiation, and American Joint Cancer Committee (AJCC) tumor stage. The PVE group had a higher percentage of patients with background cirrhosis, and the control group had a higher percentage of patients with a normal liver. As outcome measures, postoperative complications, diseasefree survival, and pattern of tumor recurrence were compared between the two groups.

\section{Statistics}

Statistical comparison between groups was performed using the chi-squared test with the Yates correction (or Fisher's exact test where appropriate) for nominal data and the Mann-Whitney $U$-test for numerical data. Disease-free survival rates were computed according to the KaplanMeier method and compared with the log-rank test. The Cox proportional hazard model was used for multivariate analysis. All analyses were performed with the statistical software SPSS (version 12; SPSS, Chicago, IL). A value of $p<0.050$ was considered statistically significant.

\section{Results}

Role of PVE and surgery

In both the resected and non-resected groups of patients, a significant increase of FRLV was noted after PVE. In the non-resected group, the median pre-embolization FRLV was $25 \%(16-37 \%)$ and $29 \%(18-46 \%)(p=0.023)$ after PVE. In the resected group, the pre-embolization FRLV was $23 \%(12-33.5 \%)$, and improved to $34 \%(20-54 \%)$ $(p=0.005)$ after PVE. One patient developed pleural 
Table 1 Baseline parameters of the portal vein embolization (PVE) group and the control group

\begin{tabular}{|c|c|c|c|}
\hline & PVE group $(n=34)$ & Control group $(n=102)$ & $p$ Value $^{\mathrm{a}}$ \\
\hline Age, years & $57(27-70)$ & $55(26-80)$ & 0.883 \\
\hline Males & $31(91 \%)$ & $78(76 \%)$ & $0.082^{\mathrm{b}}$ \\
\hline \multicolumn{4}{|l|}{ Status of non-tumorous liver in number of patients } \\
\hline Non-cirrhotic & $2(6 \%)$ & $25(25 \%)$ & 0.023 \\
\hline Chronic hepatitis & $8(24 \%)$ & $27(26 \%)$ & 0.823 \\
\hline Cirrhotic & $24(70 \%)$ & $50(49 \%)$ & 0.031 \\
\hline Alpha-fetoprotein, ng/ml & $105(2-90,400)$ & $34(1-530,600)$ & 0.645 \\
\hline Preoperative indocyanine green clearance $(\%)$ at $15 \mathrm{~min}$ & $12.5(5-28)$ & $10(3-25)$ & 0.083 \\
\hline Preoperative bilirubin, $\mu \mathrm{mol} / \mathrm{l}$ & $13(5-45)$ & $11(5-145)$ & 0.081 \\
\hline Alanine transaminase, $\mathrm{U} / \mathrm{l}$ & $57(14-316)$ & $47(11-393)$ & 0.152 \\
\hline Aspartate aminotransferase, U/l & $49(23-242)$ & $57(13-223)$ & 0.244 \\
\hline Creatinine, $\mu \mathrm{mol} / \mathrm{l}$ & $85(57-133)$ & $85(44-204)$ & 0.441 \\
\hline Platelet count, $10^{9} / \mathrm{ml}$ & $179(126-325)$ & $218(90-851)$ & 0.030 \\
\hline Albumin, $g / 1$ & $38.5(32-46)$ & $41(20-54)$ & 0.059 \\
\hline International normalized ratio & $1(0.9-1.2)$ & $1(0.8-2.6)$ & 0.035 \\
\hline Type of resection & & & 1 \\
\hline Right hepatectomy & $17(50 \%)$ & $51(50 \%)$ & \\
\hline Extended right hepatectomy & $14(41 \%)$ & $42(41 \%)$ & \\
\hline Segmentectomy & $3(9 \%)$ & $9(9 \%)$ & \\
\hline Operation duration, min & 437 (277-773) & $440(215-883)$ & 0.922 \\
\hline Operative blood loss, 1 & $0.72(0.2-4.2)$ & $0.9(0.14-6.2)$ & 0.611 \\
\hline Patients needing transfusion & $3(9 \%)$ & $11(11 \%)$ & $1.000^{\mathrm{b}}$ \\
\hline Hospital stay, days & $7(4-34)$ & $8(3-61)$ & 0.128 \\
\hline Max. tumor diameter in pathology specimen, $\mathrm{cm}$ & $7(3-17)$ & $9(3-17)$ & 0.177 \\
\hline Resection margin, $\mathrm{cm}$ & $1(0.1-3.5)$ & $1(0.1-6.5)$ & 0.023 \\
\hline Tumor cell differentiation in number of patients & & & $0.682^{\mathrm{b}}$ \\
\hline Well & $7(20 \%)$ & $17(17 \%)$ & \\
\hline Moderate & $22(65 \%)$ & $59(58 \%)$ & \\
\hline Poor & $3(9 \%)$ & $17(17 \%)$ & \\
\hline Not available & $2(6 \%)$ & $7(6 \%)$ & \\
\hline
\end{tabular}

Values are expressed as median with range unless indicated otherwise

${ }^{a}$ Mann-Whitney $U$-test, except for ${ }^{b}$ the chi-square test

effusion after PVE, which resolved spontaneously. There were no other complications reported after PVE.

Resection was not feasible in $20(37 \%)$ patients. In 10 (18.5\%) patients, increase of the FRLV was considered inadequate. In 4 of them, the decision against resection was taken during surgery despite apparently adequate FRLV on the preoperative radiological assessment. In the other 10 patients, surgery was considered not to be feasible due to (1) deteriorated liver function test results in three $(5.5 \%)$, (2) development of extrahepatic metastasis in three $(5.5 \%)$, (3) macroscopically grossly cirrhotic liver or extensive varices in two $(3.7 \%)$, (4) progression of the primary tumor in one (2\%), and (5) tumor rupture in one (Fig. 1). Thirtyfour $(63 \%)$ patients underwent curative resection. There were two $(3.7 \%)$ hospital deaths; one patient died from liver failure and the other developed sepsis and subsequent liver failure. Overall, with the combination of PVE and surgery, $32 / 54(60 \%)$ of the patients were able to achieve long-term survival (Fig. 1).

\section{Comparison of the PVE and control groups}

Postoperative complications are shown in Table 2. There were two deaths in each group. Minor complications occurred in 10/34 (29\%) of PVE patients and 25/102 (24\%) of control patients $(p=0.651)$. Major complications were seen in $6 / 34(17.6 \%)$ of PVE patients and 15/102 (14.7\%) of controls $(p=0.784)$.

The mean follow-up period in the two groups was 35 months (standard deviation, 25 months). Overall, 14 $(41 \%)$ patients in the PVE group developed recurrence, compared to $54(53 \%)$ in the control group $(p=0.322)$. 


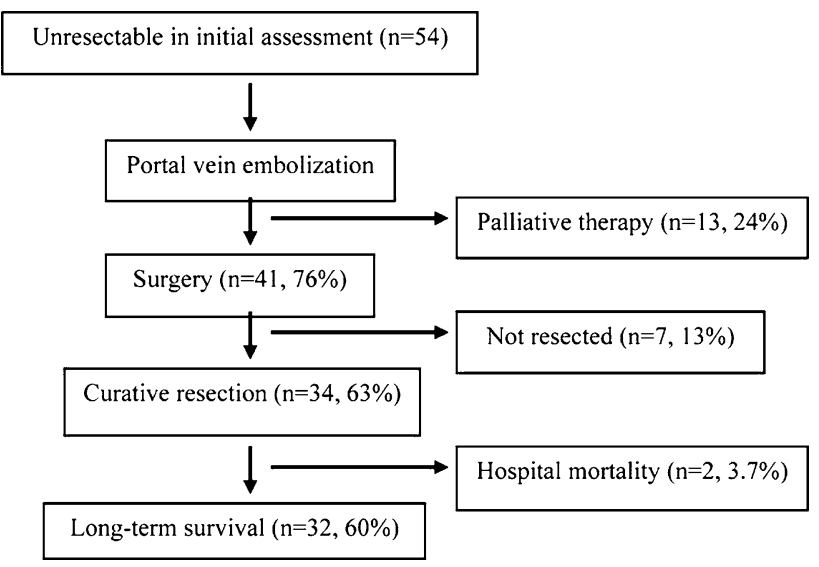

Fig. 1 Treatment flowchart of the 54 patients who underwent portal vein embolization (PVE)

Table 2 Complications in the PVE group and the control group

\begin{tabular}{llll}
\hline & $\begin{array}{l}\text { PVE group } \\
(n=34)\end{array}$ & $\begin{array}{l}\text { Control group } \\
(n=102)\end{array}$ & $p$ Value $^{\mathrm{a}}$ \\
\hline Minor complications & $10(29 \%)$ & $25(24 \%)$ & 0.651 \\
Ascites & 7 & 20 & \\
Wound infection & 2 & 5 & \\
Other & 1 & - & 0.784 \\
Major complications & $6(17.6 \%)$ & $15(14.7 \%)$ & \\
Encephalopathy & 1 & - & \\
Chest infection & 1 & 2 & \\
Postoperative bleeding & 1 & 1 & \\
Arrhythmia & - & 3 & \\
Biliary complication & - & 3 & \\
Liver failure & 1 & 3 & \\
Other & - & 1 & \\
Mortality & 2 & 2 & \\
\hline
\end{tabular}

${ }^{a}$ Chi-square test

Extrahepatic recurrence was detected in 10 (29\%) and 41 (40\%) cases in the PVE group and the control group, respectively $(p=0.310)$. Intrahepatic recurrence was detected in $10(29 \%)$ of the PVE patients and $47(46 \%)$ of the control patients $(p=0.109)$. There was no difference in the timing of detection of recurrence. Fourteen (41\%) of the recurrence cases in the PVE group and $43(42 \%)$ in the control group were detected before one year $(p=1.000)$. The median disease-free survival in the PVE group was 14 months (range: 1.9-94 months), and that in the control group was 13 months (range: 1-88 months). Figure 2 shows the disease-free survival and overall survival of the two groups. The 1-, 3-, and 5-year disease-free survival rates were 57, 29, and $26 \%$ respectively, in the control group and 60,42 , and $42 \%$, respectively, in the PVE group (log-rank, $p=0.335)$.
On multivariate Cox regression analysis, venous infiltration $(p=0.004 ; \mathrm{HR}=1.9 ; 95 \% \mathrm{CI}=1.2-3)$, largest tumor diameter $(p=0.006$; $\mathrm{HR}=1.07 ; 95 \% \mathrm{CI}=1.02$ $1.12)$, and tumor stage $(p=0.006 ; \mathrm{HR}=1.33 ; 95 \%$ $\mathrm{CI}=1.08-1.65)$ were the only individual factors associated with disease-free survival. Portal vein embolization was not a factor affecting disease-free survival $(p=0.821$; $\mathrm{HR}=1.056 ; 95 \% \mathrm{CI}=0.65-1.7$ ).

\section{Discussion}

All 54 patients who underwent PVE were not resectable initially due to inadequate FRLV. The combination of PVE and surgery was effective in $60 \%$ of these patients. Portal vein embolization was not associated with increased morbidity. Comparison of the PVE group with the controls revealed that the rates of postoperative complications, as well as the pattern of recurrence, were similar between the two groups. There was no difference in disease-free survival between the PVE group and the controls.

Because of concerns for safety and efficacy, PVE was initially limited to normal livers. In a prospective trial, Farges et al. [9] compared the operative outcomes between patients who underwent routine PVE before right hepatectomy and patients who were operated without PVE. Their study showed a clear benefit of PVE in reducing postoperative complications and kinetics of liver function in patients having background chronic liver diseases. No benefit was seen with normal livers. The group advocated routine use of PVE in these patients and further recommended liver regeneration after PVE as a marker of postoperative outcomes. Portal vein embolization has been used for cirrhotic livers with HCC in a number of other centers [10-12], although most of the reported data relate to small numbers of patients. In the present study, a higher proportion of patients in the PVE group had cirrhosis and worsened liver function, and they were expected to have poorer postoperative outcomes. However, the PVE group in fact showed statistically insignificant survival benefit. In this context, our result seems to coincide with that found by Tanaka et al. [6], who reported significantly superior survival in patients with cirrhosis.

Overall, $18.5 \%$ of our patients failed to gain adequate increase of FRLV. Twenty-four patients who underwent resection after PVE had cirrhosis. For four other cirrhotic patients who had adequate increase of FRLV, surgery was not performed because of other contraindications. This indicates that 29/44 $(66 \%)$ of the cirrhotic patients were able to achieve adequate increase of FRLV after PVE. Surgery is known to have the best results in patients with HCC [1]. The outlook for patients with unresectable HCC is bleak; their median survival is reported to be around 

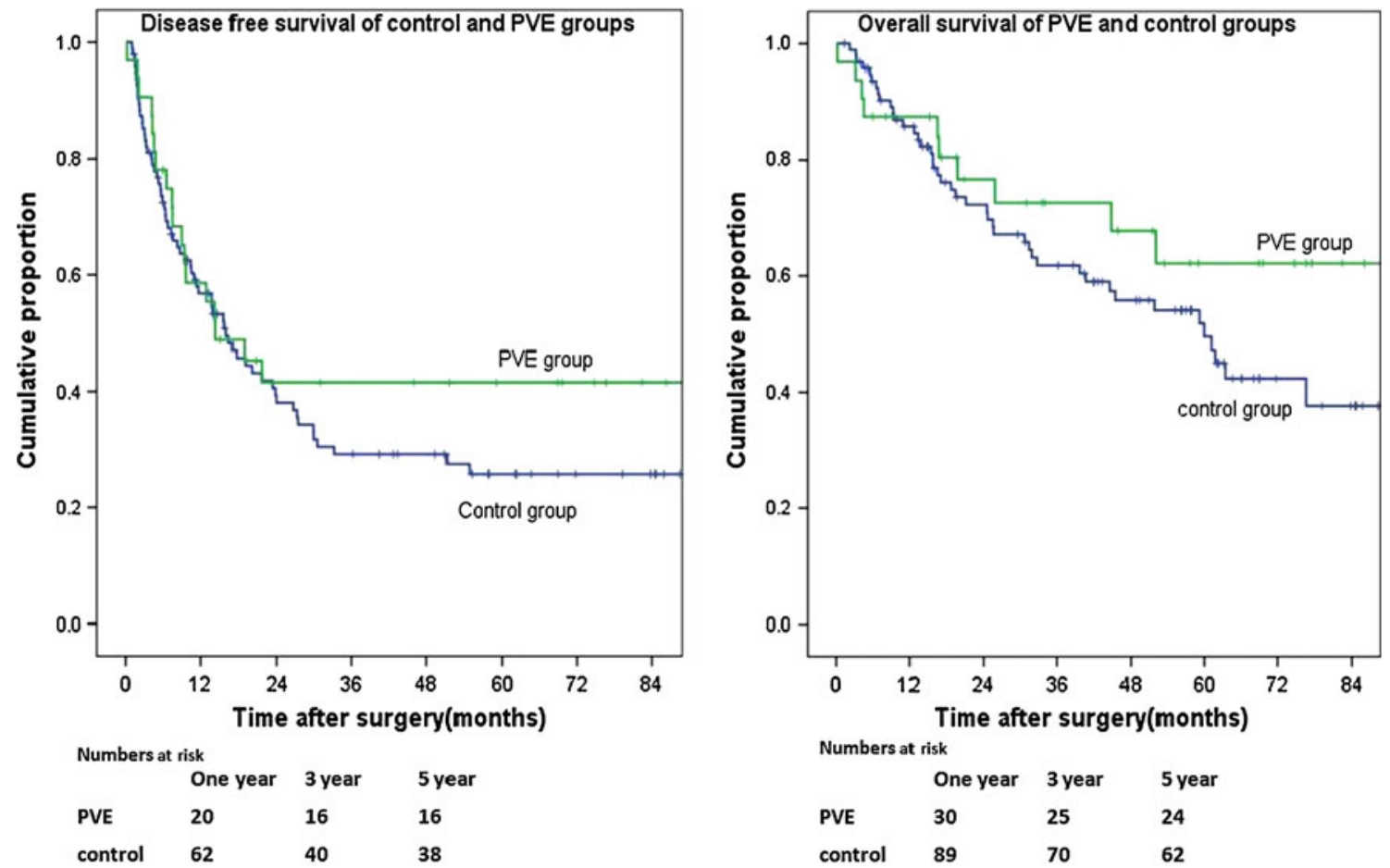

Fig. 2 Kaplan-Meier disease-free and overall survival curves of the PVE group $(n=34)$ and the control group $(n=102)$. Disease-free survival, PVE versus control: $p=0.335$; overall survival, PVE versus control: $p=0.221$ (log-rank test)

three months, and their 1-year survival could be as low as $8 \%[13,14]$. In our series, only one patient developed PVErelated minor complication. Patients in the PVE group tolerated major resection well, and postoperative adverse events were similar in the two groups. As a significant proportion of patients in the PVE group had cirrhosis and poor liver function, these results are even more significant. Similar results have been published in the past by Tanaka et al. [6] and Farges et al. [9] in particular, and both groups recommended routine administration of PVE in patients with injured livers. In view of these reports and our results, PVE should be considered an effective procedure for cirrhotic patients who have stable liver function but are denied resection because of limited FRLV. Routine administration of preoperative PVE in all cirrhotic patients appears to be effective but is beyond the results of the present study.

The risk of progression of primary tumor is a matter of debate. Hepatocellular carcinoma derives its blood supply predominantly from the hepatic artery, and embolization of the portal vein is known to alter the hepatic haemodynamics significantly. Kito et al. [15], using Doppler analysis, demonstrated a significant increase in the arterial flow of the embolized side of the liver without a significant alteration in the contralateral flow. Apart from altered haemodynamics, induction of growth factors that could influence tumor growth has been demonstrated following
PVE [16, 17]. Effects of these factors on primary tumor remain the subject of debate.

Most clinical data on tumor kinetics are based on colorectal liver metastasis [18]. These show increased tumor growth during the interval period. In our series, only one patient was found to have primary tumor progression limiting curative resection, whereas three others developed extrahepatic metastasis. In other reported studies on HCC, inoperability due to tumor progression was less than $10 \%$ $[11,19]$. With these findings taken into account, tumor progression seems a minor clinical problem.

Concurrent use of TACE has been advocated by many authors to control primary tumor and induce liver hypertrophy [20, 21]. The efficacy of this approach remains questionable [22]. We were selective in using TACE in our study group. Almost half (47\%) of our patients who had tumors likely to progress or who had a marginal liver volume were offered a combined treatment of PVE and TACE. Only one patient $(1 / 42,2 \%)$ had local tumor progression. Because of the small number of patients in our series, a subgroup analysis of our patients was not performed. However, a recent study from a Korean group compared the outcomes of 71 patients who underwent such combined treatment with the outcomes of 64 patients who had PVE alone [23]. The former group of patients were found to have a more favorable postoperative liver function. They also had better overall survival and disease-free 
survival. In a smaller but well-planned study on the combined use of PVE and TACE, in addition to better clinical outcomes and survival, pathological specimens showed complete tumor necrosis in 15 of 18 patients [24]. In the present study, the combination of PVE with TACE could have contributed to the comparable outcomes in the PVE group despite the significant proportion of cirrhotic patients. Further TACE was offered to patients who had worse tumors that were likely to become unresectable. Routine administration of PVE combined with TACE might have had a beneficial effect, although this cannot be concluded from this study.

When tumor recurrence was evaluated, we failed to demonstrate any significant difference in disease-free survival or the pattern of recurrence between the PVE and control groups. Further on multivariate analysis, only venous infiltration, tumour stage, and tumor size were shown to be associated with recurrence. To date there are at least three published series focusing on long-term recurrence of HCC after PVE. Tanaka et al. [6] evaluated 30 patients with and without PVE before surgery and showed a better overall survival in patients who had undergone PVE. However, the overall recurrence rate was not statistically different between the two groups. Palavecino et al. [25] reported disease-free survival of 84,56 , and $56 \%$ after PVE at 1, 3, and 5 years, respectively, compared to 66, 49, and $49 \%$ without PVE. Azoulay et al. [26] reported disease-free survival of 86,64 , and $21 \%$ with PVE versus 55 , 17 , and $17 \%$ without PVE at 1,3 , and 5 years, respectively.

The effect of hepatic regeneration on micrometastasis has been studied extensively. De Jong et al. [4] and Mizutani et al. [27] have shown enhanced proliferation of malignant cells in remnant livers after hepatic resection. Some consider that the same growth factors are accountable for hepatic regeneration following PVE, but others contradict this possibility and suggest that a different mechanism may be involved [28, 29]. Vascular permeation is a well-recognized tumor characteristic predicting recurrence. Based on this finding, potential benefits of portal vein obliteration ahead of surgery have been discussed by some authors [30, 31]. Transforming growth factor beta is a polypeptide that suppresses hepatocyte growth and tumor proliferation [32]. Its increased expression has been shown following PVE [33].

It is likely that multiple factors affecting tumor proliferation negatively or positively are brought on by PVE. However, all clinical data indicate that the overall effect of PVE on future recurrence is clinically insignificant. The apparently marginally better recurrence-free survival noticed in clinical studies could possibly be due to the selection of patients during PVE.

In summary, the combination of PVE and surgery was effective in $60 \%$ of the patients with initially unresectable
HCC. Two thirds of the cirrhotic patients were able to achieve a substantial increase in FRLV. After resection, the patients had rates of morbidity and recurrence-free survival comparable to those of patients who had undergone surgery without PVE. Thus, under the goal of increasing resectability, cirrhosis should not be a limiting factor against PVE for patients with stable liver function.

\section{Conflict of interest None.}

Open Access This article is distributed under the terms of the Creative Commons Attribution License which permits any use, distribution, and reproduction in any medium, provided the original author(s) and the source are credited.

\section{References}

1. Fan ST, Lo CM, Liu CL et al (1999) Hepatectomy for hepatocellular carcinoma: toward zero hospital deaths. Ann Surg 229:322-330

2. Shoup M, Gonen M, D'Angelica M et al (2003) Volumetric analysis predicts hepatic dysfunction in patients undergoing major liver resection. J Gastrointest Surg 7:325-330

3. Yokoyama Y, Nagino M, Nimura Y (2007) Mechanisms of hepatic regeneration following portal vein embolization and partial hepatectomy: a review. World J Surg 31:367-374. doi: 10.1007/s00268-006-0526-2

4. de Jong KP, Lont HE, Bijma AM et al (1995) The effect of partial hepatectomy on tumor growth in rats: in vivo and in vitro studies. Hepatology 22(4 Pt 1):1263-1272

5. Wakabayashi H, Ishimura K, Okano K et al (2001) Is preoperative portal vein embolization effective in improving prognosis after major hepatic resection in patients with advanced-stage hepatocellular carcinoma? Cancer 92:2384-2390

6. Tanaka H, Hirohashi K, Kubo S et al (2000) Preoperative portal vein embolization improves prognosis after right hepatectomy for hepatocellular carcinoma in patients with impaired hepatic function. Br J Surg 87:879-882

7. Terminology Committee of the International Hepato-PancreatoBiliary Association (2000) The Brisbane 2000 terminology of liver anatomy and resections. HPB 2:333-339

8. Dindo D, Demartines N, Clavien PA (2004) Classification of surgical complications: a new proposal with evaluation in a cohort of 6336 patients and results of a survey. Ann Surg 240:205-213

9. Farges O, Belghiti J, Kianmanesh R et al (2003) Portal vein embolization before right hepatectomy: prospective clinical trial. Ann Surg 237:208-217

10. Lee KC, Kinoshita H, Hirohashi K et al (1993) Extension of surgical indications for hepatocellular carcinoma by portal vein embolization. World J Surg 17:109-115. doi:10.1007/BF01655721

11. Yamakado K, Takeda K, Matsumara K et al (1997) Regeneration of the unembolized liver parenchyma following portal vein embolization. J Hepatol 27:871-880

12. Shimamura T, Nakajima Y, Una Y et al (1997) Efficacy and safety of preoperative percutaneous transhepatic portal embolization with absolute ethanol: a clinical study. Surgery 81:135-141

13. Llovet JM, Bustamante J, Castells A et al (1999) Natural history of untreated nonsurgical hepatocellular carcinoma: rationale for the design and evaluation of therapeutic trials. Hepatology 29:62-67

14. Yeung YP, Lo CM, Liu CL et al (2005) Natural history of untreated nonsurgical hepatocellular carcinoma. Am J Gastroenterol 100:1995-2004 
15. Kito Y, Nagino M, Nimura Y (2001) Doppler sonography of hepatic arterial blood flow velocity after percutaneous transhepatic portal vein embolization. Am J Roentgenol 176:909-912

16. Kollmar O, Corsten M, Scheuer C et al (2010) Tumour growth following portal branch ligation in an experimental model of liver metastases. Br J Surg 97:917-926

17. Kollmar O, Corsten M, Scheuer C et al (2007) Portal branch ligation induces a hepatic arterial buffer response, microvascular remodeling, normoxygenation, and cell proliferation in portal blood-deprived liver tissue. Am J Physiol Gastrointest Liver Physiol 292:G1534-G1542

18. Pamecha V, Levene A, Grillo F et al (2009) Effect of portal vein embolisation on the growth rate of colorectal liver metastases. $\mathrm{Br}$ J Cancer 100:617-622

19. Ribero D, Abdalla EK, Madoff DC et al (2007) Portal vein embolization before major hepatectomy and its effects on regeneration, resectability and outcome. Br J Surg 94:1386-1394

20. Majno PE, Adam R, Bismuth H et al (1997) Influence of preoperative transarterial lipiodol chemoembolization on resection and transplantation for hepatocellular carcinoma in patients with cirrhosis. Ann Surg 226:670-688

21. Aoki T, Imamura H, Hasegawa K et al (2004) Sequential preoperative arterial and portal venous embolizations in patients with hepatocellular carcinoma. Arch Surg 139:766-767

22. Samuel M, Chow PK, Chan Shih-Yen E et al (2009) Neoadjuvant and adjuvant therapy for surgical resection of hepatocellular carcinoma. Cochrane Database Syst Rev CD001199

23. Yoo H, Kim J, Ko G-Y et al (2011) Sequential transcatheter arterial chemoembolization and portal vein embolization versus portal vein embolization only before major hepatectomy for patients with hepatocellular carcinoma. Ann Surg Oncol 18: $1251-1257$

24. Ogata S, Belghiti J, Farges O et al (2006) Sequential arterial and portal vein embolizations before right hepatectomy in patients with cirrhosis and hepatocellular carcinoma. Br J Surg 93:10911098

25. Palavecino M, Chun YS, Madoff DC et al (2009) Major hepatic resection for hepatocellular carcinoma with or without portal vein embolization: perioperative outcome and survival. Surgery 145:399-405

26. Azoulay D, Castaing D, Krissat J et al (2000) Percutaneous portal vein embolization increases the feasibility and safety of major liver resection for hepatocellular carcinoma in injured liver. Ann Surg 232:665-672

27. Mizutani J, Hiraoka T, Yamashita R et al (1992) Promotion of hepatic metastases by liver resection in the rat. $\mathrm{Br} \mathrm{J}$ Cancer 65:794-797

28. Tashiro S (2009) Mechanism of liver regeneration after liver resection and portal vein embolization (ligation) is different? J Hepatobiliary Pancreat Surg 16:292-299

29. Takeuchi E, Nimura Y, Mizuno S et al (1996) Ligation of portal vein branch induces DNA polymerases alpha, delta, and epsilon in nonligated lobes. J Surg Res 65:15-24

30. Kinoshita H, Sakai K, Hirohashi K et al (1986) Preoperative portal vein embolization for hepatocellular carcinoma. World $\mathrm{J}$ Surg 10:803-808. doi:10.1007/BF01655244

31. Toyosaka A, Okamoto E, Mitsunobu M et al (1996) Intrahepatic metastases in hepatocellular carcinoma: evidence for spread via the portal vein as an efferent vessel. Am J Gastroenterol 91:1610 1615

32. Kusaka K, Imamura H, Tomiya $\mathrm{T}$ et al (2006) Expression of transforming growth factor-alpha and -beta in hepatic lobes after hemihepatic portal vein embolization. Dig Dis Sci 51:1404-1412

33. Moustakas A, Heldin CH (2008) Dynamic control of TGF-beta signaling and its links to the cytoskeleton. FEBS Lett 582:20512065 